\title{
Belphégor
}

Littérature populaire et culture médiatique

17 | 2019

Mutations des légitimités dans les productions culturelles contemporaines

\section{Daniel Couégnas, Fiction et culture médiatique à la Belle Epoque dans le magazine Je sais tout (1905-1914)}

\section{Clarisse Gadala}

\section{(2) OpenEdition}

\section{Journals}

Electronic version

URL: https://journals.openedition.org/belphegor/2009

DOI: $10.4000 /$ belphegor.2009

ISSN: 1499-7185

Publisher

LPCM

Electronic reference

Clarisse Gadala, "DANIEl CouÉgnas, fiction et CUlture médiatique à la belle epoque dans le MAgAZINE JE SAIS tout (1905-1914)", Belphégor [Online], 17 | 2019, Online since 16 April 2019,

connection on 25 August 2021. URL: http://journals.openedition.org/belphegor/2009 ; DOI: https:// doi.org/10.4000/belphegor.2009

This text was automatically generated on 25 August 2021.

\section{(†) $९$}

Belphégor est mis à disposition selon les termes de la Licence Creative Commons Attribution - Pas d'Utilisation Commerciale - Pas de Modification 4.0 International. 
Daniel Couégnas, Fiction et culture médiatique à la Belle Epoque dans le magazine Je sais tout (1905-1914)

Clarisse Gadala

\section{REFERENCES}

Daniel Couégnas, Fiction et culture médiatique à la Belle Epoque dans le magazine Je sais tout (1905-1914) 


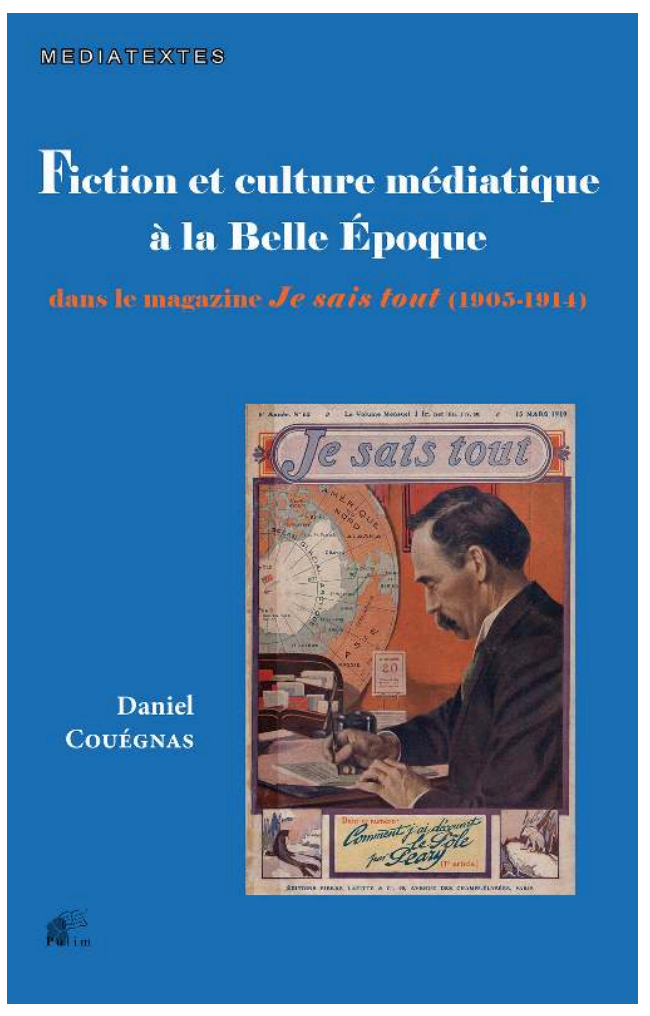

1 L'essai de Daniel Couégnas, publié en mars 2018, étudie la place de la fiction narrative dans Je Sais Tout, un magazine à vocation encyclopédique du début du XXe siècle, sans négliger de prendre en compte les autres rubriques et le contexte de publication. Il pose ainsi un regard d'ensemble critique sur les objectifs du périodique et la réalisation des ambitions de son éditeur Pierre Lafitte à une époque de développement accéléré de la culture médiatique.

Dans le premier chapitre, l'auteur contextualise la place de la fiction, et plus largement de la littérature, au sein du magazine structuré autour d'une série de rubriques dont la présence reste relativement stable au cours des dix années prises en compte dans l'étude. L'essai commence par passer en revue ces rubriques au sein desquelles se dessine une production littéraire partagée entre les mémentos, les romans et nouvelles, le théâtre, les poèmes et les articles sur la vie littéraire et critique.

3 Les mémentos, ces pages encadrées « aperçus vivants et complets des événements du mois précédent ", sont destinés à constituer une sorte d'encyclopédie de référence pour le lecteur. D'une grande importance structurelle au début de la publication (couplés aux articles dans chaque rubrique, ils constituent l'ossature du projet encyclopédique initial), ils se fondent progressivement dans le reste du magazine et sont remaniés après-guerre. A travers les échos, mondanités, anecdotes et publications du mois, ils contribuent à donner une perception de la littérature informative, associée au divertissement.

4 L'étude des autres rubriques à contenu littéraire permet à l'auteur de souligner le rôle relativement réduit accordé à la littérature dans le magazine. La fiction narrative, dans la stratégie éditoriale de Pierre Lafitte, occupe une place nouvelle dans la mesure où les œuvres inédites françaises et étrangères traduites qui sont publiées le sont le plus souvent dans leur intégralité. La périodicité mensuelle du magazine et le lectorat visé permettent cette rupture avec la structure en feuilletons et 
l'affirmation de chaque numéro comme un tout; la fidélisation du lectorat se réalise cependant aussi par le biais des séries, du type Arsène Lupin, et la publication de romans en plusieurs parties.

6 L'analyse de cette rubrique principale est ensuite associée à une revue synthétique des pièces de théâtre, en nombre non négligeable. On y trouve des comédies ou des drames, le plus souvent dans un univers mondain qui semble constituer la colonne vertébrale d'une revue tiraillée entre son ambition vulgarisatrice et un certain conformisme moral.

7 Le premier survol de la matière littéraire de Je Sais Tout, complété par deux examens rapides de la poésie et des articles de critique ou d'histoire littéraire, permet à l'auteur de dégager le constat d'une fiction de grande diffusion, à quelques exceptions près peu innovante, et prise dans les feux croisés des exigences d'une industrie culturelle naissante.

8 Le second chapitre introduit un concept développé par Edgar Morin en 1961, le "syncrétisme homogénéisé », qui s'applique au magazine selon trois axes développés successivement.

9 Le syncrétisme homogénéisé, autrement dit l'harmonisation sur le plan formel du nécessaire éclectisme des domaines abordés par un magazine qui vise le public le plus large possible, est une stratégie éditoriale que l'analyse de ses différentes manifestations littéraires permet de déceler dans Je Sais Tout.

10 Le syncrétisme des registres littéraires, tout d'abord, se traduit par la recherche d'un idéal de «littérature populaire supérieure" susceptible d'intéresser les différents segments du lectorat visé par Lafitte. Il est particulièrement à l'œuvre dans la série des Aventures d'Arsène Lupin, de Maurice Leblanc, qui occupe une place à part dans le magazine.

11 Daniel Couégnas se penche sur la genèse de ces récits, montages originaux entre un univers littéraire convenu et mondain et une intrigue populaire novatrice. Un tel contraste est symbolisé entre autres par le terme de "gentleman-cambrioleur" attribué au héros de ces aventures. Il en va de même pour d'autres auteurs qui s'insèrent parfaitement dans la politique éditoriale du magazine.

12 La proximité de la fiction narrative avec les autres formes textuelles présentes dans Je Sais Tout, par le biais d'une exploitation commune de l'actualité, est détaillée à travers plusieurs exemples. L'auteur souligne ainsi le flou entretenu entre fiction et réalité, créant ainsi les conditions d'émergence de la notion d' " hésitation d'authenticité » qui conditionne le fonctionnement des textes de la revue. Equilibre précaire mais qui, une fois obtenu, est susceptible de puissamment réactiver l'intérêt du lecteur. L'essai développe les techniques utilisées par les auteurs et les éditeurs du magazine pour créer ce jeu de miroirs, cette illusion référentielle entretenue également par les autres supports médiatiques de la revue (dessins et surtout illustrations photographiques).

13 En s'appuyant sur les notions précédemment définies et le corpus littéraire étudié ${ }^{1}$, le troisième chapitre élargit le questionnement à l'idéologie de la revue, et détaille les conceptions de la société et du monde qui transparaissent au travers des romans et nouvelles publiés dans Je Sais Tout.

14 Une première partie s'attache à la nature de la revue et détaille son idéologie. Revue apolitique et moderniste, elle utilise les ressources techniques et les met en valeur à travers une manie des données chiffrées et des comparaisons qui traduit une forme 
d'euphorie du progrès matériel et quantifiable. L'auteur montre que, soucieux de rester dans la sécurité d'un certain conformisme familial, Lafitte développe un modèle littéraire sentimental et mondain allant de pair avec l'image de la revue élégante qu'il veut donner et tenant à distance les représentants des classes les moins favorisées. Néanmoins, ce modèle lui apparaît parfois détourné, voire distancié; on voit en arrièreplan se refléter de manière assourdie les débats et inquiétudes liés aux questions sociales de l'époque.

La place du roman policier français dans la revue (et inversement, l'importance de la revue dans le développement de ce même genre littéraire) est alors étudiée à la lumière d'une fascination trouble du lectorat de la «Belle Epoque " pour le crime, doublée d'une exigence compensatoire de châtiment. Les auteurs des fictions policières semblent en effet respecter plus ou moins consciemment le cahier des charges d'une revue à la vocation familiale : à travers l'étude des différents motifs, personnages et rythmes narratifs, Daniel Couégnas montre les limites et les hésitations d'un magazine " pour tous ». Ce schéma se répète lorsque Je Sais Tout voit ses fictions prendre place audelà des frontières hexagonales. La perception sourde de l'étranger comme menace, identifiée comme propre à la bourgeoisie de l'époque, est doublée d'une conscience aiguë de la supériorité de la "race blanche » lisible notamment dans les fictions qui se déroulent en Afrique et mettent en scène une imagerie répétitive et des jugements en clichés. Il en découle l'impression d'une forme d'autisme culturel de la part du périodique, qui se contente de présenter à ses lecteurs un reflet valorisant et sécurisant d'eux-mêmes.

Complétant l'étude des fictions déjà abordées sous d'autres angles dans les pages précédentes, le quatrième chapitre se focalise sur le récit d'aventures policières. Pourquoi, mis à part quelques récits de Conan Doyle et de Gaston Leroux et les apparitions récurrentes d'Arsène Lupin, le reste du corpus a-t-il sombré dans l'oubli ? L'auteur étudie méthodiquement les différents aspects de fictions dont les hésitations et les ambiguïtés reflètent celles d'un magazine qui se veut à la fois populaire et mondain. Il fait le constat d'un modèle policier français inabouti, dont la mise en scène privilégie les milieux bourgeois (afin de se démarquer du roman-feuilleton à la connotation trop populaire) et fait la part belle à une thématique sentimentale sans doute destinée à attirer la part féminine du lectorat. Tiraillés entre ces injonctions contradictoires, les récits pèchent en outre par une trame trop volatile, détruisant l'intérêt dramatique initial, ou par l'absence d'un personnage d'enquêteur dans lequel le lecteur puisse se projeter.

Par contraste, les aventures d'Arsène Lupin, auxquelles l'auteur consacre une importante sous-partie, prennent une réjouissante distance dans un récit ironique et théâtral qui questionne l'origine du discours et relativise l'énonciation. Sans minimiser la place des marqueurs propres à l'époque, l'auteur montre comment le récit détourne les règles et les codes à son profit, devenant ainsi lisible à plusieurs niveaux. De même, l'analyse du Fauteuil hanté, roman de Gaston Leroux publié sous forme de feuilleton dans le journal de novembre 1909 à avril 1910, montre la particularité d'un récit qui rompt avec la narration convenue des autres fictions policières par un véritable jeu sur l'énonciation. L'attention accordée au langage, notamment, le goût du paradoxe et la maitrise de procédés d'accroche déstabilisent le lecteur et contribuent à la production d'un véritable "intérêt romanesque ", suscitant en permanence la curiosité et la jubilation. Daniel Couegnas fait donc le constat d'une grande hétérogénéité du corpus 
policier, handicapé par le mélange de genres difficilement conciliables qu'entraîne la mise en place d'un syncrétisme consubstantiel au projet de l'éditeur de Je Sais Tout.

Le cinquième et dernier chapitre s'intéresse enfin de plus près au contenu scientifique de la fiction narrative dans le périodique. Comment le progrès technologique et la science sont-ils mis en scène dans les récits? Quels thèmes sont abordés? Et que dit leur traitement sur l'image de la société telle que le magazine se la représente et l'offre à ses lecteurs? L'auteur traite en quatre sous-parties la vision de Je Sais Tout. De manière prédictible, « l'homme qui sait » est au centre de la majorité des fictions, qu'il soit savant, ingénieur ou possède simplement la connaissance nécessaire au moment voulu. Daniel Couégnas passe en revue les différents modèles proposés, souvent porteurs d'une forme d'optimisme rassurant qui peut même parfois tendre au messianisme scientifique avec par exemple la célébration de l'image du grand savant. Le magazine se fait alors l'écho des crises et des débats contemporains via l'introduction de motifs littéraires récurrents qui diffusent les découvertes du temps.

19 Cette célébration du progrès technologique (aéroplane, automobile, télégraphe sans fil) n'est pas exempte d'une certaine distance, comme le montre l'analyse de fictions qui portent un regard humoristique ou satirique sur ces découvertes. Quelques dissonances pessimistes se font même sentir : de manière plutôt exceptionnelle, puisque l'auteur ne recense que sept fictions faisant état d'un dysfonctionnement de type catastrophiste, mais réelle. Cependant, l'étude de ces cas permet à Daniel Couégnas de conclure que ces exceptions confirment en quelque sorte la règle de la normalité; le dénouement toujours heureux, grâce à l'intervention des savants, fait de l'état catastrophique une parenthèse maîtrisée symbole d'un optimisme affiché en règle d'or de l'industrie médiatique. Les débats entre sceptiques et clairvoyants, comme autant de topoi littéraires contribuent à la légèreté lénifiante d'un magazine de grande diffusion.

Le chapitre se termine sur une dernière forme de connaissance scientifique exploitée dans les fictions du périodique. L'émergence du domaine de la psychopathologie, croisé avec les sciences expérimentales, permet aux romanciers de mettre en scène des formes psychiques spectaculaires (qu'il s'agisse de génie, de folie ou de crime). Hérédité, dégénérescence et contagion dans les fictions métaphorisent les doutes de l'époque et laissent sourdre une forme d'angoisse derrière le masque lisse de l'imagerie mondaine.

21 Quels sont pour conclure les apports de Je Sais Tout? Pour Daniel Couégnas, il ne s'agit pas véritablement d'un magazine de vulgarisation scientifique: les connaissances proposées font trop souvent appel à l'irrationnel, et sont biaisées par les clichés qui les accompagnent. L'encyclopédisme affiché au départ s'avère donc être en réalité un éclectisme qui propose au lecteur une réalité nuancée de rêve, un univers amusant et bizarre, d'où le dérangeant et le polémique sont exclus. Le filtre posé par son éditeur Lafitte laisse cependant passer certaines inquiétudes et angoisses de la Belle Epoque, à l'image d'une société hantée par un scepticisme identitaire. 


\section{NOTES}

1. L'essai est suivi d'une liste des romans et nouvelles étudiés.

\section{AUTHOR}

\section{CLARISSE GADALA}

Conservatrice de Bibliothèque, Clarisse Gadala est responsable du fonds patrimonial au Centre national de la littérature pour la jeunesse (CNLJ) de la Bibliothèque nationale de France. 